\title{
MODELLING COUPLED ELECTRIC FIELD AND MOTION OF BEAM OF IONIC POLYMER-METAL COMPOSITE
}

\author{
Ireneusz DOMINIK*, Filip KASZUBA*, Janusz KWAŚNIEWSKI*
}

\begin{abstract}
*AGH University of Science and Technology, Faculty of Mechanical Engineering and Robotics, Department of Process Control
\end{abstract}
dominik@agh.edu.pl, fkaszuba@agh.edu.pl,kwa_@@agh.edu.pl

\begin{abstract}
In this paper, a mathematical model of electromechanical transduction of lonic Polymer-Metal Composites is presented. The aim of the research was to create a physics-based, geometrically scalable model to use in control systems. The relation between actuating voltage and the tip displacement was described with a transfer function. The model is derived from the basic physical properties of researched materials. To calculate the final transfer function, two impedance models are considered - with and without neglecting the resistance of the metal electrodes. In this paper, the model with non-zero electrode resistance is calculated. Later, the model is simplified (taking the physical properties into account) and the numerical values based on the parameters of the samples are calculated. The simplifications allow the model to predict the response to low-frequency sine wave actuation. The frequency-domain characteristics of the samples were created experimentally and compared to the model. The results have proven the accuracy of the model.
\end{abstract}

Key words: Ionic Polymer-Metal Composite, Mathematical Model, Smart Materials

\section{INTRODUCTION}

The lonic Polymer-Metal Composites (IMPC) are a class of smart materials, which can be used in various technical applications. They can be applied as actuators, sensors, energy generators or self-sensing materials.

The IPMC can operate in two modes - sensing and actuating. The material consists of two layers of noble metal and ionconducting polymer between them. The material is also filled with particles of a polar solvent (i.e. water), which create clusters that move freely inside the polymer layer (Shahinpoor and Kim, 2001). Such a composite is capable of bending with great strain. The applied voltage causes the cations with attached solvent particles to gather near the negative potential. This causes a local increase in density and bends the strip towards the positive electrode.

Such materials have numerous applications in automation, mechatronics, biomedical sciences, military science and entertainment (Pugal et al., 2010). A reverse effect is also present mechanical deformation causes electrical voltage to appear on the electrodes. In this case, the material can be used as an energy generator, e.g. in energy harvesting systems (Aureli et al., 2010) or displacement sensor (Bahramzadeh and Shahinpoor, 2011). Actuation model will be presented in this paper.

\section{DERIVATION OF THE MODEL OF IPMC}

Presented model is a generalization of the work of Farinholt, (2005); Sia Nemat-Nasser and Li (2000), utilizing a surface resistance model and Laplace transform representation. The resulting transfer functions in their precise, nonlinear form have very high computational complexity, thus they should be reduced to a lower order linear transfer function to enable the use in control systems.
Tab. 1. Symbols, their values, units and descriptions (Chen and Tan, 2008)

\begin{tabular}{|c|c|c|c|}
\hline Symbol & Value & Unit & Description \\
\hline$F$ & 96487 & $\mathrm{C} / \mathrm{mol}$ & Faraday constant \\
\hline$R$ & 3.3143 & $\mathrm{~J} /(\mathrm{mol} \cdot \mathrm{K})$ & Gas constant \\
\hline$R_{p}^{\prime}$ & 0.37 & $\Omega m$ & $\begin{array}{l}\text { Resistivity across } \\
\text { the IPMC ( } \mathrm{x} \text { axis) }\end{array}$ \\
\hline$Y$ & $5.71 \cdot 10^{8}$ & $\mathrm{~Pa}$ & Young's modulus \\
\hline$r_{1}^{\prime}$ & 22.3 & $\Omega m$ & $\begin{array}{l}\text { Resistivity of the } \\
\text { electrode in z direction }\end{array}$ \\
\hline$r_{2}^{\prime}$ & $1.8 \cdot 10^{-5}$ & $\Omega m$ & $\begin{array}{l}\text { Resistivity of the } \\
\text { electrode in } x \text { direction }\end{array}$ \\
\hline$d$ & $1.38 \cdot 10^{-9}$ & $\mathrm{~m}^{2} / \mathrm{s}$ & $\begin{array}{c}\text { Ion diffusion } \\
\text { coefficient }\end{array}$ \\
\hline$C^{-}$ & 1091 & $\mathrm{~mol} / \mathrm{m}^{3}$ & Anion concentration \\
\hline$K_{e}$ & $1.34 \cdot 10^{-6}$ & $\mathrm{~F} / \mathrm{m}$ & $\begin{array}{c}\text { Effective dielectric } \\
\text { constant of the poly- } \\
\text { mer }\end{array}$ \\
\hline$\alpha_{0}$ & 0.129 & $\mathrm{~J} / \mathrm{C}$ & $\begin{array}{l}\text { Stress-charge density } \\
\text { correlation coefficient }\end{array}$ \\
\hline$\omega_{n}$ & 28.9 & $1 / \mathrm{s}$ & $\begin{array}{c}\text { Angular frequency } \\
\text { of undamped beam } \\
\text { oscillations }\end{array}$ \\
\hline$\zeta$ & 0.1 & - & $\begin{array}{c}\text { Damping ratio } \\
\text { of beam oscillations }\end{array}$ \\
\hline$T$ & 300 & $\mathrm{~K}$ & Temperature \\
\hline$W$ & 0.01 & $\mathrm{~m}$ & Width of the sample \\
\hline$L$ & 0.04 & $\mathrm{~m}$ & Length of the sample \\
\hline$h$ & 200 & $\mu \mathrm{m}$ & $\begin{array}{l}\text { Thickness of the } \\
\text { sample }\end{array}$ \\
\hline
\end{tabular}

The derivation of the model is based on the work of (Chen and Tan, 2008), with a different simplification method. The model was later tested experimentally. 


\subsection{Partial differential equations}

Following equations were primarily presented by(Farinholt, 2005). Distribution of charges in the polymer layer is governed by equations(1) and (2).

$$
\begin{aligned}
& \mathrm{E}=\frac{\mathrm{D}}{\kappa_{\mathrm{e}}}=-\nabla \cdot \emptyset \\
& \nabla \cdot \mathrm{D}=\rho=\mathrm{F} \cdot\left(\mathrm{C}^{+}-\mathrm{C}^{-}\right)
\end{aligned}
$$

where: D - electrical displacement, $\mathrm{E}$ - electric field, $\varnothing$ - electric potential, $\rho$ - charge density, $\mathrm{C}^{+}$- cation concentration.

Later, following the Farinholt's calculations we get a partial differential equation (3), that models the spatial and temporal variation in charge density due to an initial charge distribution.

$\frac{\partial \rho}{\partial \mathrm{t}}-\mathrm{d} \frac{\partial^{2} \rho}{\partial \mathrm{x}^{2}}+\frac{\mathrm{F}^{2} \mathrm{dC}^{-}}{\kappa_{\mathrm{e}} \mathrm{RT}}\left(1-\mathrm{C}^{-} \Delta \mathrm{V}\right) \rho=0$

\subsection{Electrical impedance model}

Based on an assumption made by Nemat-Nasser and Li (Sia Nemat-Nasser and Li, 2000), that stress $\sigma$ is proportional to the charge density $\rho$ and stress-charge density correlation coefficient $\alpha_{0}$

$$
\sigma=\alpha_{0} \rho
$$

the electrical impedance model will be used in the creation of control-oriented model. The value of the stress-charge density correlation coefficient $\alpha_{0}$ depends on the effective dielectric constant of the composite and geometrical parameters of the ion clusters inside an ionomeric layer (S Nemat-Nasser and Li, 2000).

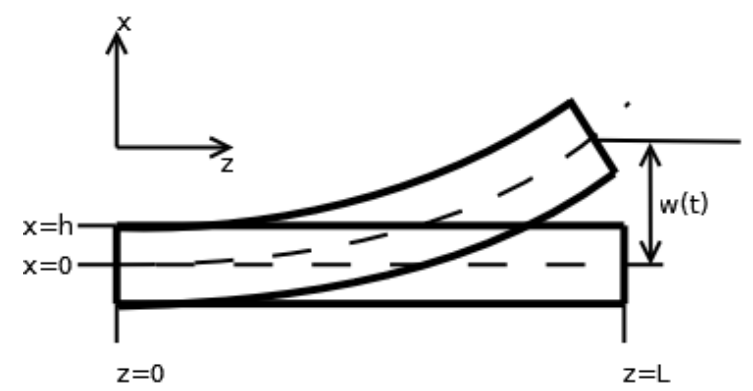

Fig. 1. Geometrical model of IPMC cantilever beam

To simplify the notation, the constants are aggregated into following equation (5):

$K \triangleq \frac{F^{2} d C^{-}}{\kappa_{e} R T}\left(1-C^{-} \Delta V\right)$

Later, a Laplace transform is applied to equation (3), taking into account that $\rho$ is independent from $\mathrm{y}$.

$s \rho(x, z, s)-d \frac{\partial^{2} \rho(x, z, s)}{\partial x^{2}}+K \rho(x, z, s)=0$

Next, $\beta^{2}(s)=(s+K) / d$ is defined. Assuming a symmetrical charge distribution in y axis, a general solution of equation (6) is presented in equation (7).

$\rho(x, z, s)=2 c_{2}(z, s) \sinh (\beta(s) x)$ where $c_{2}(z, s)$ depends on boundary values of PDE (3). Based on equations (7), (1) and (2), electric field (8) and potential (9) can be calculated:

$E(x, z, s)=2 c_{2}(z, s) \frac{\cosh (\beta(s) x)}{\kappa_{e} \beta^{2}(s)}-a_{1}(z, s)$

$\varnothing(x, z, s)=-2 c_{2}(z, s) \frac{\sinh (\beta(s) x)}{\kappa_{e} \beta^{2}(s)}$

$-a_{1}(z, s) x+a_{2}(z, s)$

Two cases of boundary conditions are considered - with and without consideration of electrode resistance. In both cases relations can be found between tip displacement and applied voltage.

\subsection{Model without the resistance of the electrodes}

In this case, the electrodes of the polymer are considered to be ideal conductors. Electric potential is constant throughout the two surfaces and equals:

$\emptyset( \pm h, z, s)=\frac{ \pm V(s)}{2}$

Next, the constants of equations (8) and (9) are calculated from equation (6), and the value of electric force is calculated:

$E(h, z, s)=-\frac{V(s)}{2 h} \frac{\gamma(s)(s+K)}{s \gamma(s)+K \tanh (\gamma(s))}$

where: $\gamma(s)=\beta(s) h$. Charge is calculated by an integral of the electrical displacement $\mathrm{D}(12)$.

$$
\begin{aligned}
Q(s)=\int_{0}^{W} \int_{0}^{L} D & (h, z, s) d z d y \\
& =\int_{0}^{W} \int_{0}^{L} \kappa_{e} E(h, z, s) d z d y
\end{aligned}
$$

The impedance of the IPMC is derived from:

$Z_{1}(s)=\frac{V(s)}{s Q(s)}=\frac{s+K(\tanh (\gamma(s) / \gamma(\mathrm{s}))}{C s(s+K)}$

where: $\mathrm{C}=\kappa_{\mathrm{e}} \mathrm{WL} / 2 \mathrm{~h}$, and is considered to be the capacitance of the composite.

\subsection{Model with the resistance of the electrodes}

Surface of the electrode of the IPMC consists of nanoparticles that are created during chemical reduction of salts of noble metals such as platinum. This has some influence on the surface resistance of the IPMC, which has to be taken into the model. Equations (14) and (15) describe the relations between $i_{s}(z, s)$, $i_{k}(z, s), i_{p}(z, s)$ and $\emptyset_{ \pm}(z, s)$.

$\frac{\partial \emptyset_{ \pm}(z, s)}{\partial z}=\mp \frac{r_{1}{ }^{\prime}}{W} i_{s}(z, s)$

$\frac{\partial i_{s}(z, s)}{\partial z}=-\left(i_{p}(z, s)+i_{k}(z, s)\right)$

For $z=0, \quad \emptyset_{ \pm}(z, s)= \pm V(s) / 2$, thus boundary conditions can be calculated from(16)

$\emptyset( \pm h, z, s)=\emptyset_{ \pm}(z, s) \mp i_{p}(z, s) r_{2}{ }^{\prime} / W$ 


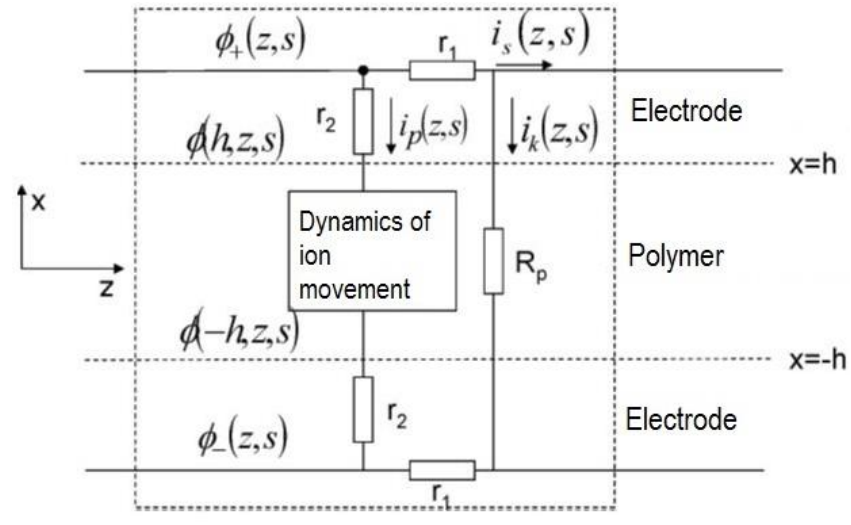

Fig. 2. Model of the IPMC with surface resistance (Chen and Tan, 2008)

By using (14) and (16), we get:

$\varnothing( \pm h, z, s)=\frac{ \pm V(s)}{2} \mp \int_{0}^{z} \frac{r_{1}{ }^{\prime}}{W} i_{s}(\tau, s) d \tau-\frac{r_{2}{ }^{\prime}}{W} i_{p}(z, s)$

Unknowns from the general equation (9) can be now calculated. The key assumption of modelling IPMC is that the ions do not pass between polymer and metal, thus total ion flux for $\mathrm{x}=\mathrm{h}$ and $\mathrm{x}=-\mathrm{h}$ equals zero $(18)$.

$\left(\frac{\partial^{3} \emptyset}{\partial x^{3}}-\frac{F^{2} C^{-}}{\kappa_{e} R T}\left(1-C^{-} \Delta V\right) \frac{\partial \emptyset}{\partial x}\right)_{x= \pm h}=0$

Based on (17) and (11), while taking the boundary condition (18) into account, the electric force in this case is:

$\mathrm{E}(h, z, s)=\frac{\emptyset(x, z, s)}{h} \frac{\gamma(s)(s+K)}{s \gamma(s)+K \tanh (\gamma(s))}$

The current in the negative part of the $\mathrm{x}$ axis is defined as positive. Considering the ion movement,

$i_{p}(z, s)=-s W D(h, z, s)=-s W \kappa_{e} E(h, z, s)$

and

$i_{k}(z, s)=\frac{\emptyset_{+}(z, s)-\emptyset_{-}(z, s)}{\frac{R p^{\prime}}{W}}$

Using the equations (19), (20) and (21), the differential equation (16) can be solved for $\mathrm{i}_{\mathrm{s}}(\mathrm{z}, \mathrm{s})$ with a boundary condition $\mathrm{i}_{\mathrm{s}}(\mathrm{L}, \mathrm{s})=0$. Total current $\mathrm{I}(\mathrm{s})=\mathrm{i}_{\mathrm{s}}(0, \mathrm{~s})$, thus the transfer function for the impedance is presented in (22):

$Z_{2}(s)=\frac{V(s)}{I(s)}=\frac{2 \sqrt{B(s)}}{A(s) \tanh (\sqrt{B(s)} L)}$

where:

$A(s)=\frac{\theta(s)}{\left(1+\frac{r^{\prime}{ }^{2} \theta(s)}{W}\right)}+\frac{2 W}{R_{p}^{\prime} p}$

$B(s)=\frac{r_{1}^{\prime}}{W} A(s)$

$\theta(s)=\frac{s W \kappa_{e} \gamma(s)(s+K)}{h(s \gamma(s)+K \tanh (\gamma(s)))}$

It should be noted, that $\mathrm{Z}_{2}(\mathrm{~s})$ is consistent with $\mathrm{Z}_{1}(\mathrm{~s})$ forr $r_{1}^{\prime} \rightarrow 0, r_{2}^{\prime} \rightarrow 0$ and $\mathrm{R}_{\mathrm{p}}^{\prime} \rightarrow \infty$.

\subsection{The transfer function of the IPMC cantilever beam}

The model was later developed to suit the movement of cantilever beam.Firstly, the transfer function $\mathrm{H}(\mathrm{s})$ will be calculated. It describes the connections between the tip displacement $\mathrm{w}(\mathrm{L}, \mathrm{s})$ and actuating voltage $\mathrm{V}(\mathrm{s})$, while ignoring the mechanical properties of the beam itself. Equation (26), describes the stress $\sigma(\mathrm{x}, \mathrm{z}, \mathrm{s})$, based on assumption (4):

$\sigma(x, z, s)=2 \alpha_{0} c_{2}(z, s) \sinh (\beta(s) x)$

The constant $c_{2}(z, s)$ is calculated from the impedance model. While considering the model with surface resistance, we get the bending moment:

$M(z, s)=\int_{-h}^{h} x \sigma(x, z, s) W d x=$

$=\int_{-h}^{h} 2 \alpha_{0} W x c_{2}(z, s) \sinh (\beta(s) x) d x=$

$=-\frac{2 \alpha_{0} W K \kappa_{e}(\gamma(s)-\tanh (\gamma(s))) \emptyset(h, z, s)}{(s \gamma(s)+K \tanh (\gamma(s)))}$

It is assumed that an axial force does not appear and the charge density distribution is described by equation (7). From the linear beam theory(Mohammadpour et al. 2012) and equation (17):

$$
\begin{gathered}
\frac{\partial^{2} w(z, s)}{\partial z^{2}}=\frac{M(z, s)}{Y I} \\
=-\frac{2 \alpha_{0} W K \kappa_{e}(\gamma(s)-\tanh (\gamma(s))) \emptyset(h, z, s)}{Y I(s \gamma(s)+K \tanh (\gamma(s)))}= \\
=-\frac{\alpha_{0} W K \kappa_{e}(\gamma(s)-\tanh (\gamma(s)))}{2 Y I(s \gamma(s)+K \tanh (\gamma(s)))} \cdot \frac{V(s)-2 \int_{0}^{z r^{\prime}}{ }_{1} i_{s}(\tau, s) d \tau}{1+\frac{r^{\prime}{ }^{2} \theta(s)}{W}},
\end{gathered}
$$

where: $\mathrm{Y}$ is Young's modulus of the beam, and $\mathrm{I}=2 \mathrm{Wh}^{3} / 3$ is a secondary moment of area for the cross section of the beam. When above equation is solved with boundary conditions $w(0, s)=0$ andw $(0, s)=0$, we get:

$$
\begin{gathered}
w(L, s)= \\
=-\frac{\alpha_{0} W K \kappa_{e}(\gamma(s)-\tanh (\gamma(s)))}{2 Y I(s \gamma(s)+K \tanh (\gamma(s)))} \cdot \frac{V(s) L^{2}-4 \int_{0}^{L} \int_{0}^{z} \int_{0}^{z{ }^{\prime}} \frac{r^{\prime}}{W} i_{s}(\tau, s) d \tau d z d z \prime}{1+\frac{r^{\prime}{ }_{2} \theta(s)}{W}}
\end{gathered}
$$

Based on these calculations, we get:

$V(s) L^{2}-4 \int_{0}^{L} \int_{0}^{z} \int_{0}^{z^{\prime}} \frac{r_{1}^{\prime}}{W} i_{s}(\tau, s) d \tau d z d z^{\prime}=2 L^{2} X(s) V(s)$

where:

$X(s)=-\frac{1-\operatorname{sech}(\sqrt{B(s)} L)-\tanh (\sqrt{B(s)} L \sqrt{B(s)} L)}{B(s) L^{2}}$.

Finally, the transfer function $H(s)=\frac{w(L, s)}{V(s)}$ of the IPMC is given by equation (32):

$H(s)=-\frac{L^{2} \alpha_{0} W K \kappa_{e}(\gamma(s)-\tanh (\gamma(s)))}{2 Y I(s \gamma(s)+K \tanh (\gamma(s)))} \cdot \frac{2 X(s)}{1+\frac{r^{\prime}{ }_{2} \theta(s)}{W}}$

To make a complete description of the behaviour of the IMPC, the $H(s)$ transfer function has to be used in serial connection with the transfer function, which describes the mechanical properties 
if the beam. The beam is considered to be a second order oscillatory system, described by transfer function (33):

$G(s)=\frac{\omega_{n}^{2}}{s^{2}+2 \xi \omega_{n} s+\omega_{n}^{2}}$

The final transfer function is given by:

$G_{f}(s)=H(s) \cdot G(s)$

\subsection{Simplification of the transfer function}

The transfer function presented in equations (32) and (33) describe the behaviour of the polymer accurately, but is far too complicated to be implemented in e.g. control systems. Therefore, the function will be simplified using mathematical transformations and values of physical properties from table 1 . The values that could not be measured were taken from previous author's work (Kwaśniewski and Dominik, 2011; Chen and Tan, 2008). First, the transfer function $H(s)$ is split into three following transfer functions:

$H(s)=f(s) \cdot g(s) \cdot X(s)$

where:

$f(s)=-\frac{L^{2} \alpha_{0} W K \kappa_{e}(\gamma(s)-\tanh (\gamma(s)))}{2 Y I(s \gamma(s)+K \tanh (\gamma(s)))}$

$g(s)=\frac{2}{1+\frac{r^{\prime}{ }^{2} \theta(s)}{W}}$

Based on the knowledge of physical properties of the samples, it is noticed, that $|\gamma(\mathrm{s})| \gg 10 \mathrm{i} \mathrm{K} \gg 10^{6}$. It is also assumed, that for low frequencies $(<100 \mathrm{~Hz}) \tanh (\gamma(s)) \approx 1$. This allows to substitute the function $\gamma(\mathrm{s})$ with a constant $\gamma$. $\gamma(s) \approx h \sqrt{\frac{K}{d}}=\gamma$

This leads to following approximate solutions:

$$
\begin{aligned}
& f(s) \approx-\frac{L^{2} \alpha_{0} W K \kappa_{e}(\gamma-1)}{2 Y I(s \gamma+K)} \\
& \theta(s) \approx \frac{s W K \kappa_{e} \gamma(s+K)}{h(s \gamma+K)} \\
& g(s) \approx \frac{2 h(\gamma s+K)}{r^{\prime}{ }_{2} \gamma \kappa_{e} s(s+K)+h(\gamma s+K)}
\end{aligned}
$$

The Taylor series approximation of sinh and cosh functions is used to simplify $X(s)$ part of the transfer function.

$X(s) \approx \frac{1+\sum_{n=0}^{m}\left(\frac{a^{2 n+2}}{(2 n+1) !}-\frac{a^{2 n}}{2 n !}\right)}{\sum_{n=0}^{m} \frac{a^{2 n+2}}{2 n !}}$,

where: $a=\sqrt{B(s)} L$.

This approximation works well for low-frequency responses of the beam. Such approximated model is still a physics-based model, so it is geometrically scalable.

\section{NUMERICAL VALUES OF THE MODEL AND EXPERIMENTAL RESULTS}

Testing of the model was performed on laboratory samples of ionic polymer-metal composites. The sample was working in a cantilever beam setup, and its tip displacement was meas- ured. The actuating signal was generated by National Instruments NI9263 card.

Due to low current values $(10 \mathrm{~mA})$ generated by the card, avoltage follower was based on an operational amplifier was used as a current amplifier to power the composite. Displacement measurements were performed by laser sensor. Measurements from the laser sensor were acquired by National Instruments 9215 card. Voltage supplied to the sample was also measured, to calculate the phase offset.Data acquisition and signal generation was controlled by a PC based system with LabView software. The whole measuring circuit is shown in Fig. 3.

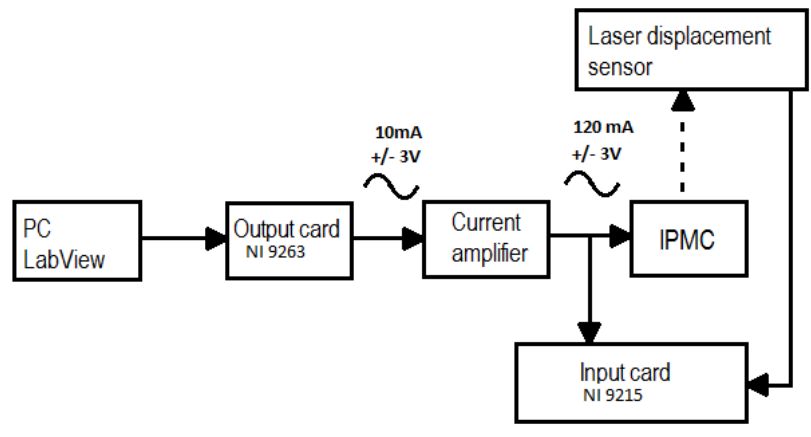

Fig. 3. Block diagram of the research stand

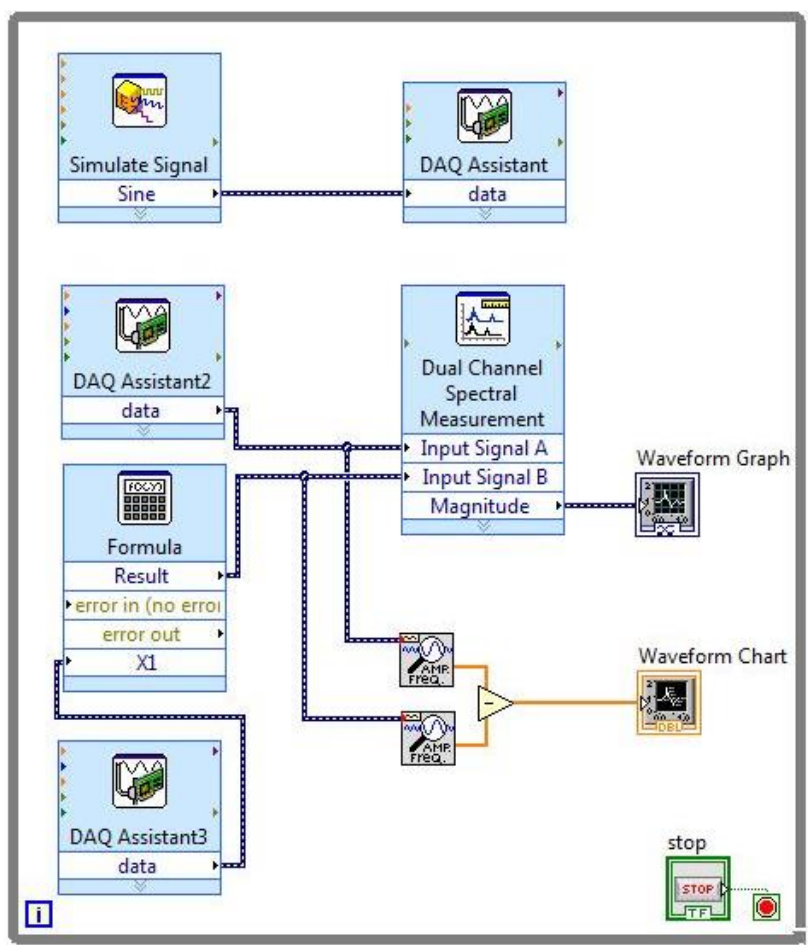

Fig. 4. Signal generation, data acquisition and calculations of magnitude and phase offset in LabView

\subsection{Numerical values of transfer functions}

After substitution of numerical values to equations (39) and (41):

$f(s)=\frac{-2099}{1,103 \cdot 10^{4} s+4,194 \cdot 10^{6}}$ 
$g(s)=\frac{4,41 s+1677}{2,69 \cdot 10^{-7} s^{2}+2,205 s+838,7}$

The calculation of $X(s)$ is based on solutions of equations (23), (24), (25) and $a=\sqrt{B(s)} L$. Second order Taylor polynomial is used. The final form of $H(s)$ transfer function consists of 45 th order polynomials in nominator and denominator, with coefficients ranging from $10^{-83}$ to $10^{97}$. Due to its complexity, the transfer function was simplified by using reduce function from Robust Control Toolbox of MATLAB software, which produced a following transferfunction, describing the electrochemical processes behind the actuation:

$H(s)=\frac{-0,01413 s+0,0954}{s^{2}+322,3 s+236,4}$

The second part of the transfer function, describing the mechanical properties of the beam, is calculated using the parameters from table 1.

$G(s)=\frac{835}{s^{2}+5,78 s+835}$

The final transfer function, which relates the tip displacement with actuating voltage is given in equation (47)

$P(s)=H(s) \cdot G(s)=$

$\frac{-11,8 s+79,66}{s^{4}+328,1 s^{3}+2934 s^{2}+2,705 \cdot 10^{5} s+1,974 \cdot 10^{5}}$

\subsection{Time-domain analysis of the model}

The lonic Polymer-Metal Composites are known to respond more predictably to AC actuation(Nemat-Nasser, 2002). DC response depends on number of factors, such as time from the last actuation, hydration, time of continuous actuation etc. Previous studies (Kwaśniewski and Dominik, 2011) have shown, that DC actuation of the IPMC is a strongly nonlinear and time-varying phenomenon, therefore cannot be described by Laplace transfer function.

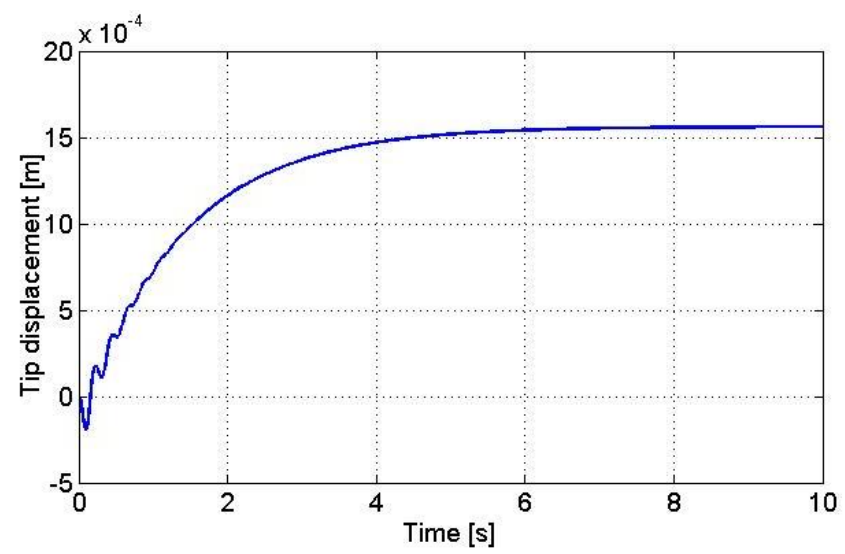

Fig. 5. Step response of the transfer function $\mathrm{P}(\mathrm{s})$ (obtained from Eq.47)

This facts, and the assumptions made in section 2.6 of this paper prove, that the described model cannot be used to predict the step response of the IPMC. Step responses of the model (Fig. 5) and the sample (Fig. 6.) to the actuation voltage of 3,78V.

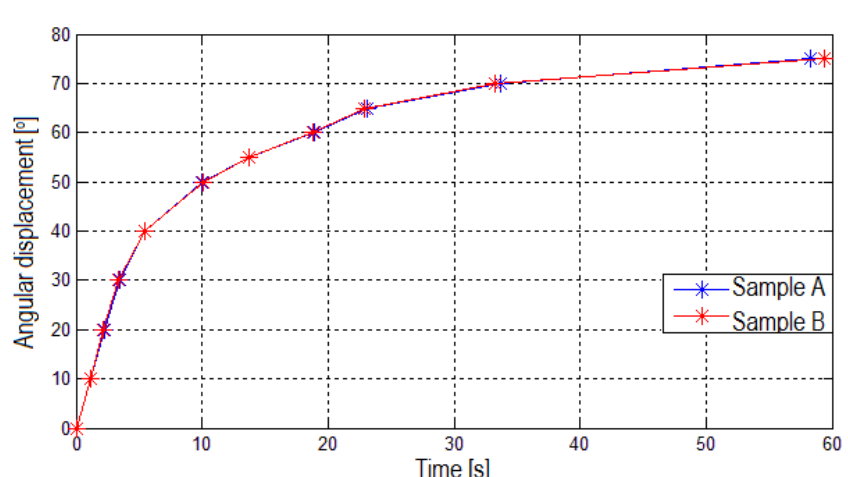

Fig. 6. Step response of the samples

\subsection{Frequency-domain analysis of the model}

The Bode plot (Fig. 3) of the transfer function (47) was plotted using MATLAB. The observed peak indicates the resonance frequency of the cantilever beam.

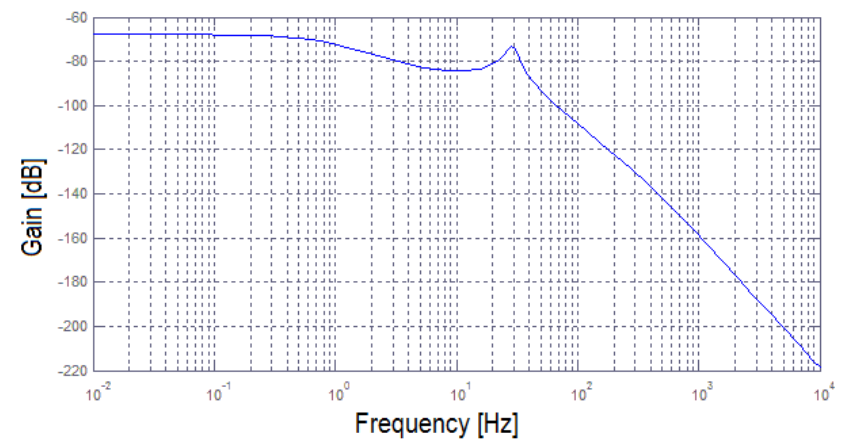

Fig.7. Bode magnitude plot of the transfer function $\mathrm{P}(\mathrm{s})$ (from Eq.47)

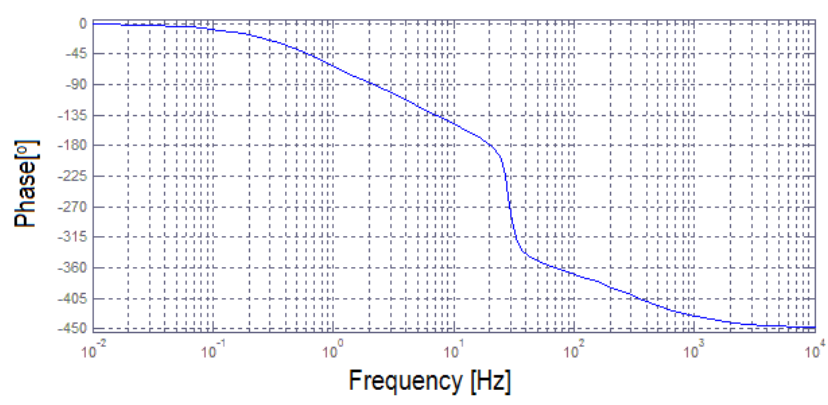

Fig. 8. Bode phase plot of the transfer function $\mathrm{P}(\mathrm{s})$ (from Eq.47)

\subsection{Experimental frequency-domain analysis of the samples}

The samples of the IPMC were mounted horizontally to compensate for gravity. The sample was placed in front of an angular displacement scale for visual inspection of the movement and different experiments, conducted with image acquisition system.

The frequency-domain analysis was conducted by measuring the response to a sine wave voltage signal with different frequencies, varying from 0,1 to $1000 \mathrm{~Hz}$. Three repetitions were conducted for each frequency and two samples were tested. The results presented here are mean values from the three repetitions. Both gain and phase shift were calculated online by LabView software 
(Fig. 4).Comparison of frequency domain plots of the mode and measurements is presented in the figure 10. Values of the gain of the sample for $1000 \mathrm{~Hz}$ was lower than the level of noise generated by the laser sensor, so the values in the figure are approximate.

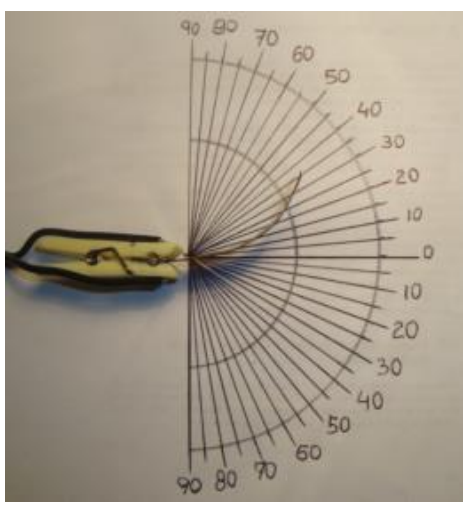

Fig. 9. Mounted IPMC sample during actuation

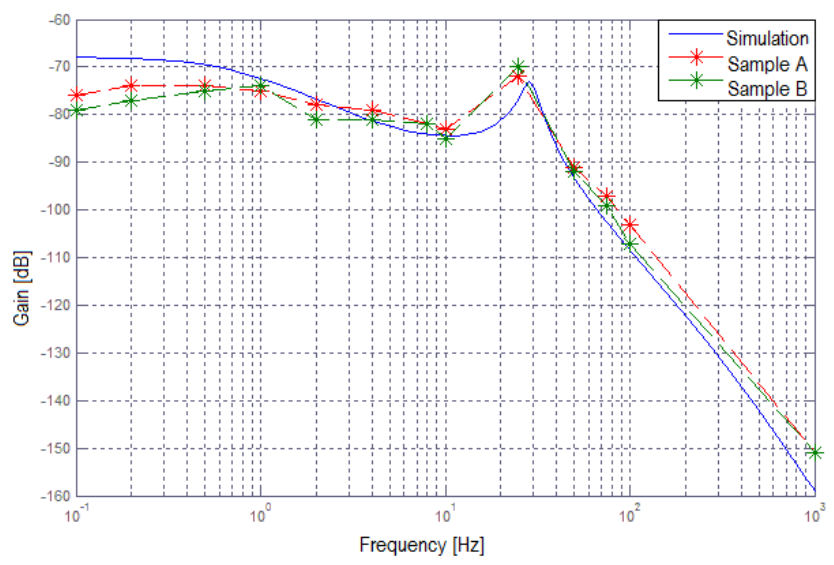

Fig. 10a. Comparison of Bode magnitude plots of the model simulation and the samples

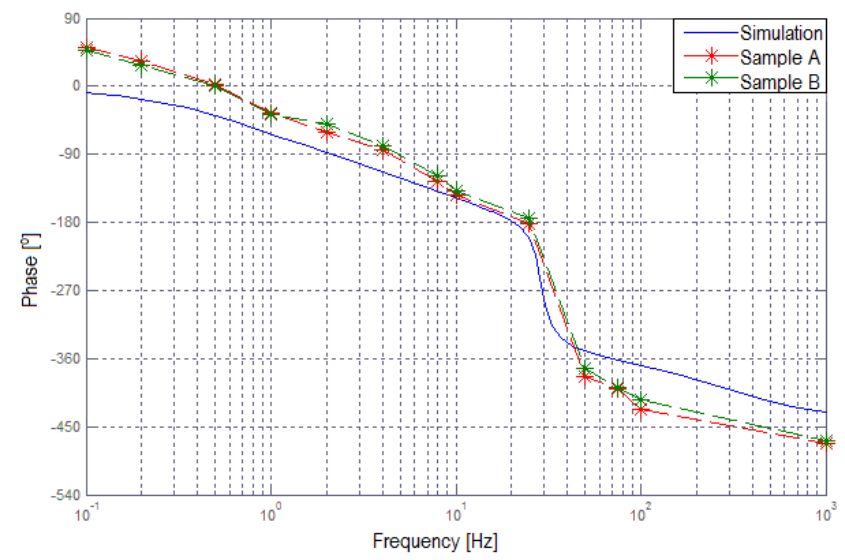

Fig. 10b. Comparison of Bode phase plots of the model simulation and the samples

\section{CONCLUSIONS}

The mathematical model of electromechanical transduction of lonic Polymer-Metal Composites was presented in the paper. The aim of the research was to create a physics-based, geometri- cally scalable model to use in control systems. The relation between actuating voltage and the tip displacement was described with a transfer function. The model was derived from the basic physical properties of researched materials.

As seen in figure 10, the model is fitted very well to the experimental data. The error of about $5 \%$ can be caused by strong nonlinearities of the IPMC actuators and simplification of the transfer functions. Furthermore, the samples are highly sensitive to hydration and change their properties with time. The experimental value of mechanical resonance peak (ca. $30 \mathrm{~Hz}$ ) is consistent with assumed parameters, which proofs the quality of the model. To calculate the final transfer function, two impedance models were considered - with and without neglecting the resistance of the metal electrodes. Additionally, the model with nonzero electrode resistance was calculated. Later, the model was simplified (taking the physical properties into account) and the numerical values based on the parameters of the samples were calculated. The simplifications allow the model predict the response to low-frequency sine wave actuation. The frequencydomain characteristics of the samples were created experimentally and compared to the model. The results have proven the accuracy of the model.

Further research will focus on developing the presented in the paper model into the model which will describe the time response to $\mathrm{DC}$ voltage actuation.

\section{REFERENCES}

1. Aureli M., Prince C., Porfiri M., Peterson S. D. (2010), Energy harvesting from base excitation of ionic polymer metal composites in fluid environments, Smart Materials and Structures, 19(1), 15003.

2. Bahramzadeh Y., Shahinpoor M. (2011), Dynamic curvature sensing employing ionic-polymer-metal composite sensors, Smart Materials and Structures, 20(9), 94011.

3. Chen Z., Tan, X. (2008), A Control-Oriented and Physics-Based Model for lonic Polymer--Metal Composite Actuators, Mechatronics, IEEE/ASME Transactions on, 13(5), 519-529.

4. Farinholt K. M. (2005), Modeling of dynamic behaviors of ionic polymer transducers for sensing and actuation, Virginia Polytechnic Institute.

5. Kwaśniewski J., Dominik I. (2011), Laboratory Research on Mechanical Features of lonic Polymer Metal Composite IPMC, Acta Mechanica et Automatica, 5(3), 65-72 (in polish)

6. Nemat-Nasser S. (2002), Micromechanics of actuation of ionic polymer-metal composites, Journal of Applied Physics, 92(5), 2899.

7. Nemat-Nasser S., Li J. (2000a), Electromechanical response of ionic polymer-metal composites, Journal of Applied Physics, 87, 3321-3331.

8. Nemat-Nasser S., Li J. Y. (2000b), Electromechanical response of ionic polymer-metal composites, Journal of Applied Physics, 87(7), 3321.

9. Pugal D., Jung K., Aabloo A., Kim, K. J. (2010), lonic polymermetal composite mechanoelectrical transduction: review and perspectives, Polymer International, John Wiley \& Sons, Ltd., 59(3), 279-289.

10. Shahinpoor M., Kim K. J. (2001), lonic polymer-metal composites: I. Fundamentals, Smart Materials and Structures, 10(4), 819.

Acknowledgement: The research work was supported by the Polish government from the funds dedicated to statutory activities of the Department of Process Control at AGH University of Science and Technology in Krakow, Poland. 